\title{
Molecular dynamics study of orientational order and rotational melting in clusters of $\mathrm{TeF}_{6}$
}

\author{
Shimin Xu, Lawrence S. Bartell \\ Department of Chemistry, University of Michigan, Ann Arbor, MI 48109, USA (FAX: +1-313-747-4865)
}

Received: 22. November 1993

\begin{abstract}
Molecular dynamics simulations of the behavior of molecules in crystalline clusters of $\mathrm{TeF}_{6}$ were carried out on systems of $100,150,250$, and 350 molecules. Several diagnostic functions were applied to investigate whether rotational melting occurred before translational melting. These functions included the coefficient of rotational diffusion $D_{\theta}(T)$, the "orientational Lindemann index" $\delta_{\theta}(T)$, the "orientational angular distribution function" $Q(\theta, T)$, and the "orientational pair-correlation function" $g_{\theta}(r, T)$. All indicators implied that rotational melting occurred before translational melting, that it began with the outermost molecules, and that its onset for smaller clusters was at lower temperatures than for larger clusters. Results also showed that the rotational transition coincided with the transition from a lower symmetry phase (monoclinic) to cubic, a phenomenon that had been noted by others to occur with some regularity for systems of globular molecules.
\end{abstract}

PACS: $36.40 ; 64.60 . \mathrm{Qb} ; 82.20 . \mathrm{Wt}$

\section{Introduction}

Molecular clusters have proven to be particularly fruitful subjects to study in a continuing program of research on the dynamics of phase changes in condensed matter. Hexafluorides, whose highly symmetric molecules pack in a rich variety of stable and metastable solid phases, provide attractive examples to investigate. The warmest solid phase for all but those of the heaviest metals is a well-studied, plastically crystalline phase whose intriguing properties remain a popular subject for research [1]. Moreover, the time evolution of phase changes of condensed hexafluorides can be readily followed by electron diffraction analyses of cluster beams cooling in supersonic flow [2]. Parameters characterizing the intermolecular interaction functions for hexafluorides are fairly well established, making their systems amenable to mo- lecular dynamics (MD) simulations. Remarkably, it turns out that some of the same transitions which can be followed experimentally on a microsecond time scale by electron diffraction can be studied computationally, in colder clusters, by molecular dynamics on a sub-picosecond time scale. A number of illuminating MD investigations of clusters and bulk systems of $\mathrm{SF}_{6}$ by Pawley and coworkers have already appeared [3-15].

Of the hexafluorides, $\mathrm{TeF}_{6}$ first received disproportionate attention in our program because of its apparently anomalous behavior [16]. Although the seeming anomalies are now well-understood [17-19], the system displayed properties that made it and its close relatives, $\mathrm{SeF}_{6}$, $\mathrm{MoF}_{6}$, and $\mathrm{WF}_{6}$ particularly favorable subjects for $\mathrm{MD}$ investigations of structure and transformation. In a prior paper [19] in this series we investigated the relative stabilities of five distinct crystalline phases of $\mathrm{TeF}_{6}$ (of the over one-dozen solid phases being analyzed currently [20]) and the size-dependence of melting points and other phase transitions of clusters. In the present work we sought to find whether crystalline clusters of the quasispherical molecule $\mathrm{TeF}_{6}$ exhibit the phenomenon of rotational melting prior to translational melting. Such behavior had been reported for a number of systems of globular molecules, including adamantane, neopentane, and methane [21]. It had been said in these cases to take place concurrently with a transition from a phase of lower symmetry to a cubic phase.

In addition to the techniques conventionally applied to the diagnosis of phases and phase changes in the many reported studies of atomic clusters [22,23], we invoke several others related specifically to molecular orientations. Considerations of orientation are obviously crucial to the aims of the present paper but, of course, have no relevance for atomic clusters. Perhaps the most natural function to try is the coefficient of rotational diffusion, $D_{\theta}$, determined from the rate of increase of mean-square angular displacements of molecules. We also test three new functions introduced in an earlier paper in this series [24] to find their suitability for the purpose at hand. These functions include the "orientational Lindemann index" 
$\delta_{\theta}$, the "orientational angular distribution function" $Q(\theta)$, and the "orientational pair-correlation function" $g_{\theta}(r)$. The first of these is loosely analogous to the conventional Lindemann index $\delta$ [22], and describes the rootmean-square amplitudes of angular displacements of molecules relative to each other. The second function is a normalized histogram of angles $\left(\theta_{\alpha \beta}\right)_{i j}$ between bonds $\alpha$ on molecule $i$ and bonds $\beta$ on molecule $j$. The histogram incorporates all $\alpha, i, \beta$, and $j$, and is in some degree the orientational analog of the conventional angular distribution function $P(\theta)$ [23]. The third quantity reduces to the conventional pair-correlation function $g(r)$ when all molecules have identical orientations. When molecules at distance $r$ differ in orientation, however, the amplitude of $g_{\theta}(r)$ is diminished in proportion to the square of the difference. Explicit definitions of the coefficient of diffusion and the three new functions are given in the appendix.

\section{Procedure}

Computations were carried out, as described in detail elsewhere $[19,24]$, with a modified version of the program MDMPOL [25], using a seven-site model potential function based on neutral atom-atom interactions characterized fully in earlier papers in this series [16, 19, 24]. Clusters containing $100,150,250$, and 350 molecules of $\mathrm{TeF}_{6}$ were prepared by constructing approximately spherical aggregates having bcc structures, and cooling them in 10 deg stages of 5000 timesteps of $10 \mathrm{fs}$ each $(1000$ timesteps for equilibration followed by 4000 over which statistics were accumulated). The clusters all transformed to single crystals of the well-ordered monoclinic phase [19] in the vicinity of $80-100 \mathrm{~K}$. After they had been cooled to $10 \mathrm{~K}$ without undergoing any further change of phase, the monoclinic clusters were heated until they transformed and ultimately melted, again at a rate of $10 \mathrm{deg}$ every 5000 timesteps. The various indicators of orientational behavior were applied to find whether any signs of change occurred that could be considered to correspond to rotational melting.

It was necessary to formulate plausible criteria to enable inferences to be drawn about rotational melting from the diagnostic functions. To initiate the analysis we assumed that rotational diffusion implied melting when $D_{\theta}$ exceeded $0.01 \mathrm{rad}^{2} / \mathrm{ps}\left(10^{10} \mathrm{rad}^{2} / \mathrm{s}\right)$. This value corresponded to rotational mean-square displacements of $0.03 \AA^{2} / \mathrm{ps}$ for the fluorine atoms at the ends of the $1.81 \AA$ bonds in $\mathrm{TeF}_{6}$ making the tangential mean-square displacements numerically equal to the linear mean-square displacements already found appropriate for translational melting. Such a correspondence between threshold values for rotational and translational melting had been introduced in an earlier study of carbon tetrachloride [26] where rotational and translational melting were found to occur more or less together. In that work the recognition of rotational melting was guided by considerations of rotational entropy and signal-to noise ratio.

Although the coefficient of diffusion is, perhaps, one of the most fundamental indicators of whether a material is fluid or not, diffusion is by its nature noisy, even when averaged (as it is here) over many independent time origins. The standard deviation in the displacement of a single random walker is of the same magnitude as the displacement, itself, independent of the number of steps it has undertaken. Therefore, there is some merit in examining alternative signatures of melting using functions that are intrinsically less noisy, including the three new functions. Since they are less direct measures of fluidity, however, they need to be calibrated. In the following we examine whether the various functions, when calibrated against $D_{\theta}$ for one cluster size, can give mutually consistent results for all clusters. Specifically, we consider the temperature dependence of the function $\delta_{\theta}$, of the value of $Q(\theta)$ at $\theta=90^{\circ}$, and of the heights of the first peak of $g_{\theta}(r)$. For comparison, the corresponding value of the conventional pair-correlation function $g(r)$ will also be examined.

\section{Results}

As clusters are heated, their rotational diffusion coefficients and rotational Lindemann indices increase steadily, as shown in Figs. 1 and 2. It is clear that the onset of rotational diffusion occurs earlier, the smaller the cluster. It is instructive to examine how the rotational freedom of a molecule depends upon its position in a cluster. To
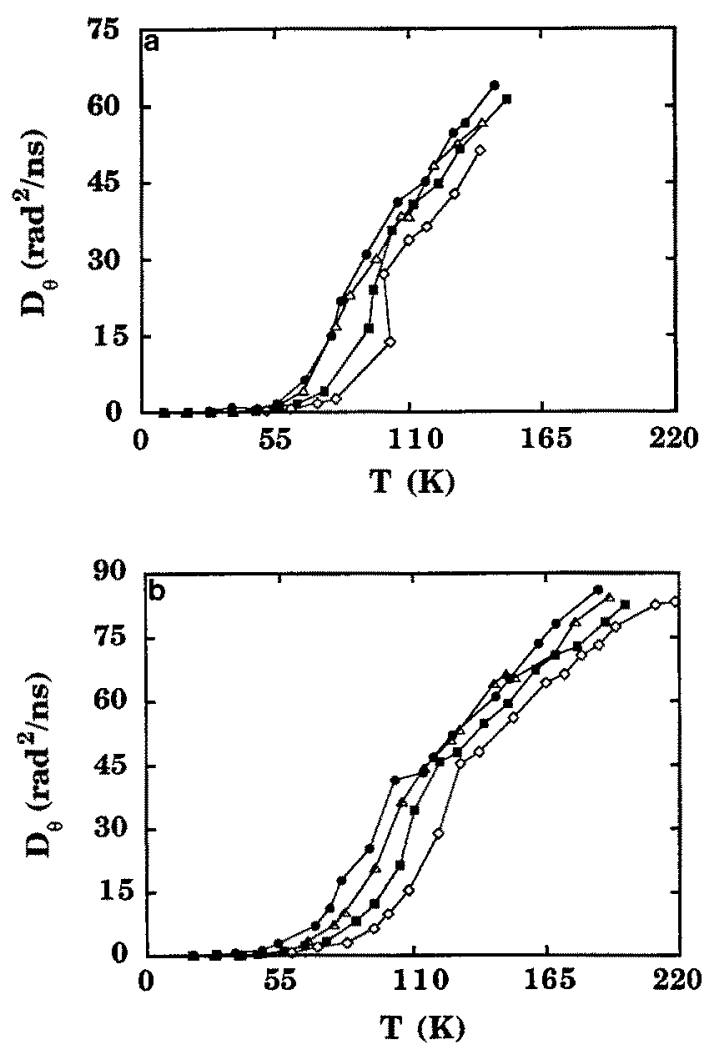

Fig. 1. Rotational diffusion coefficients $D_{\theta}$ averaged over all molecules of a given $N$-molecule cluster as a function of the temperature in cooling $\mathbf{a}$, and heating $\mathbf{b}$ runs. Filled circles, $N=100$; triangles, $N=150 ;$ filled squares, $N=250 ;$ diamonds, $N=350$ 

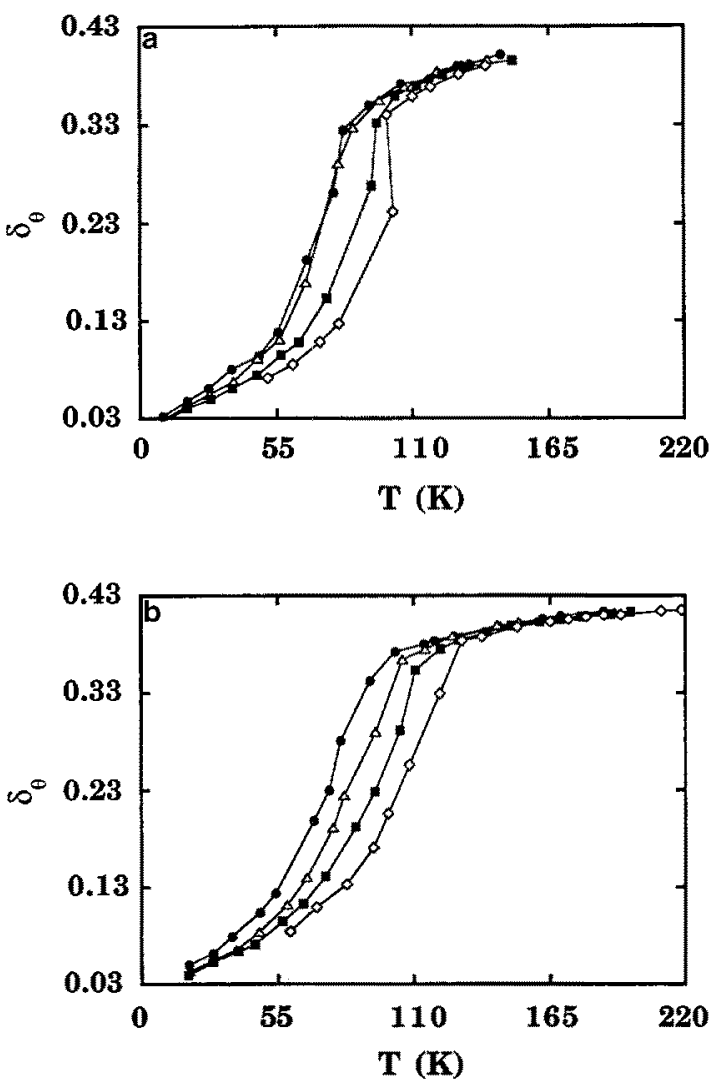

Fig. 2. Orientational Lindemann indices $\delta_{\theta}$ averaged over all molecules of a given $N$-molecule cluster as a function of the temperature in cooling a, and heating b runs. Filled circles, $N=100$; triangles, $N=150 ;$ filled squares, $N=250$; diamonds, $N=350$

do this, we divided clusters into different "shells" of molecules, defined for each cluster to correspond to the regions of the peaks of the pair correlation function of Te atoms in the monoclinic phase. Placing dividing lines at the minima between the peaks, we found four, five, six, and seven shells in the 100-, 150-, 250-, and 350-molecule clusters. For the 150-molecule cluster, for example, the shells, from inner to outer, contain $15,12,32,49$, and 42 molecules.

As can be seen in Fig. 3, the outermost molecules in this cluster rotate much more freely at low temperatures than do the molecules in the core. For this reason the disproportionate activity at the cluster surface makes the arithmetic mean of $D_{\theta}$ over the entire cluster a poor gauge of whether the cluster as a whole has melted, rotationally. Of course, because the melting of small molecular aggregates is diffuse, being spread over a temperature range of many degrees, it is arbitrary to assign a definite melting point to a cluster as a whole. Nevertheless, it is reasonable to define a characteristic temperature for the transition of the cluster by following the convention proposed previously for translational melting [19]. This can be done unambiguously, once a threshold has been adopted, by applying the criterion to individual molecules. The characteristic temperature can be taken to be the temperature at which half the molecules in the cluster are in "rotationally melted states", namely those with values of $D_{\theta}$
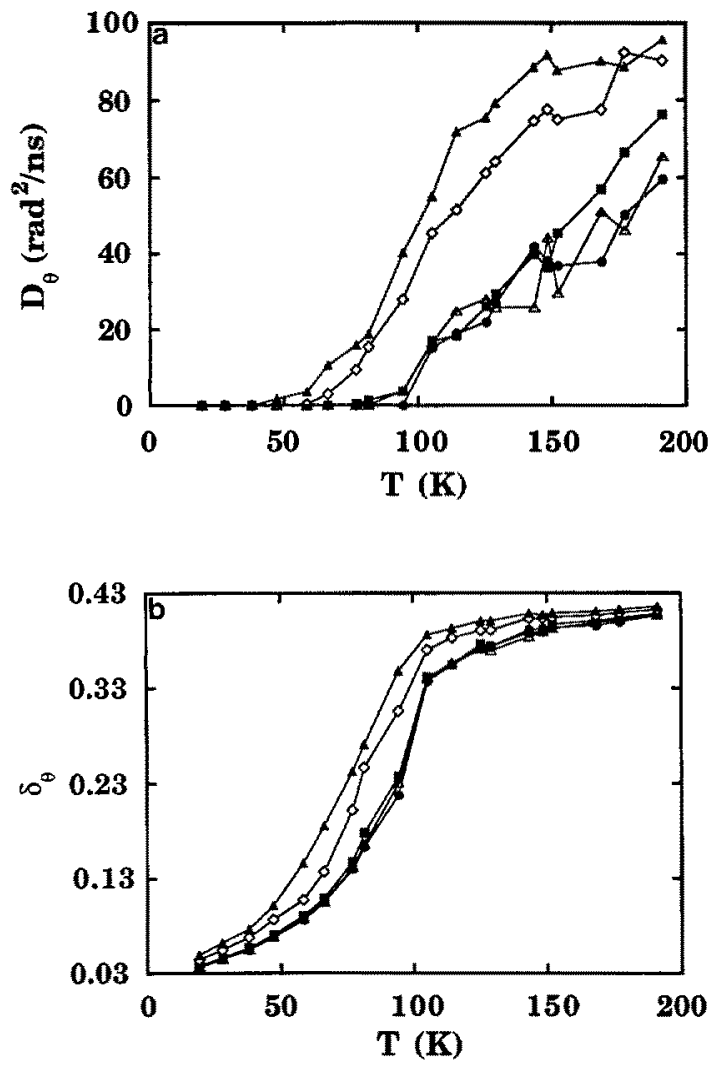

Fig. 3. a Rotational diffusion coefficients $D_{\theta}$ and $\mathbf{b}$ orientational Lindemann indices $\delta_{\theta}$, averaged over all of the molecules in individual shells of the 150 -molecule cluster in a cooling run. Filled circles, innermost shell 1 ; triangles, shell 2 ; filled squares, shell 3 ; diamonds, shell 4; filled triangles, shell 5

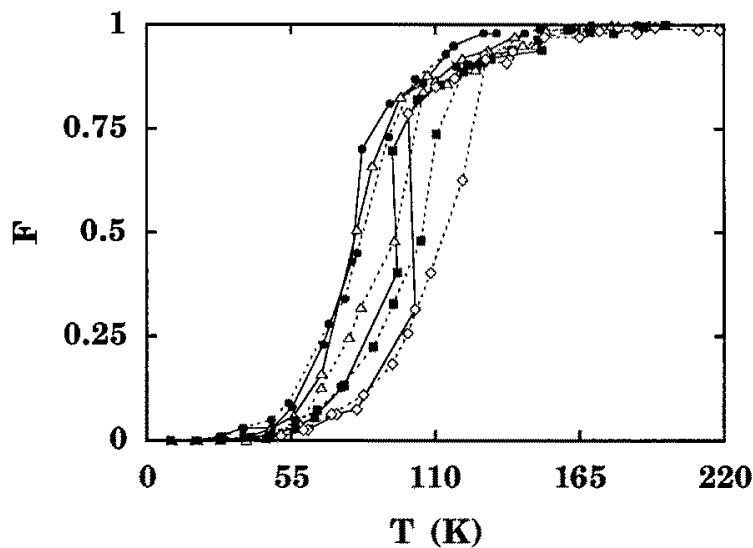

Fig. 4. Temperature dependence of the fractions of $N$-molecule clusters that are considered to be melted according to the coefficients of diffusion of the individual molecules. Solid curves correspond to cooling runs, dashed, to heating runs. Filled circles, $N=100 ;$ triangles, $N=150 ;$ filled squares, $N=250 ;$ diamonds, $N=350$

exceeding $0.01 \mathrm{rad}^{2} / \mathrm{ps}$. Figure 4 illustrates for various clusters how $F$, the molten fraction of each cluster, depends upon the temperature according to this convention.

The function $\delta_{\theta}(T)$ can be seen in Fig. 3 to be substantially less noisy and more moderate in its site to site 


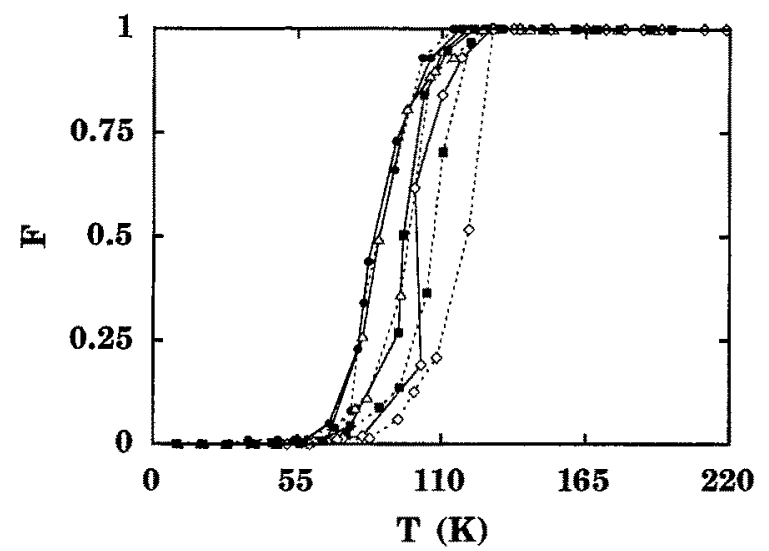

Fig. 5. Temperature dependence of the fractions of $N$-molecule clusters that are considered to be melted according to the orientational Lindemann indices of the individual molecules. Solid curves correspond to cooling runs, dashed, to heating runs. Filled circles, $N=100$; triangles, $N=150$; filled squares, $N=250$; diamonds, $N=350$

variation than is $D_{\theta}(T)$. In these respects, $\delta_{\theta}$ and $D_{\theta}$ behave in the same way as their translational counterparts, $\delta$ and $D_{r}$. If a threshold value of 0.33 for rotational melting is assigned to $\delta_{\theta}$, results are consistent with those of $D_{\theta}$. Figure 5 depicts the temperature dependence of the corresponding fraction $F$ of molecules in rotationally melted states. Because of the moderate properties of $\delta_{\theta}$, the average value over the entire cluster (taken to be 0.33 ) yields a criterion for rotational melting that is virtually the same as that of $F(T)$ obtained from an analysis of the individual molecules.

Alternative diagnoses of rotational behavior can be constructed from the functions $Q(\theta)$ and $g_{\theta}(r)$. The value of the orientational angular distribution at $90^{\circ}$ depends strongly upon the orientational order of molecules in the clusters. This order can be seen in Fig. 6 to decline markedly beyond a certain temperature. If the value 0.013 is taken as the threshold value of $Q\left(90^{\circ}\right)$ corresponding to rotational melting, results are compatible with those from $D_{\theta}$ and $\delta_{\theta}$.

Heights of peaks of the orientational pair-correlation $g_{\theta}(r)$ systematically damp more completely, the greater is the misfit in orientation between pairs of molecules separated by a distance of $r$. A given pair of bonds, one from each molecule, contributes to $g_{\theta}(r)$ an amount that decays as a Gaussian function of the difference between the two bond directions. Figure 7 illustrates the result when the first peak of $g_{\theta}(r)$ is selected, using a standard deviation of $5^{\circ}$ for the Gaussian damping. Of course, the conventional pair-correlation function $g(r)$ of Fig. 8 also damps as thermal motions increase. Nevertheless, the effect of the transition is considerably more substantial in $g_{\theta}(r)$. Choosing the value of 0.3 (in Fig. 7) to represent the threshold for rotational melting yields results consistent with the other criteria. A summary comparing the results of the five different criteria for rotational melting is presented in Table 1.

Because all of the above criteria for rotational melting are somewhat arbitrary, it is desirable to determine how
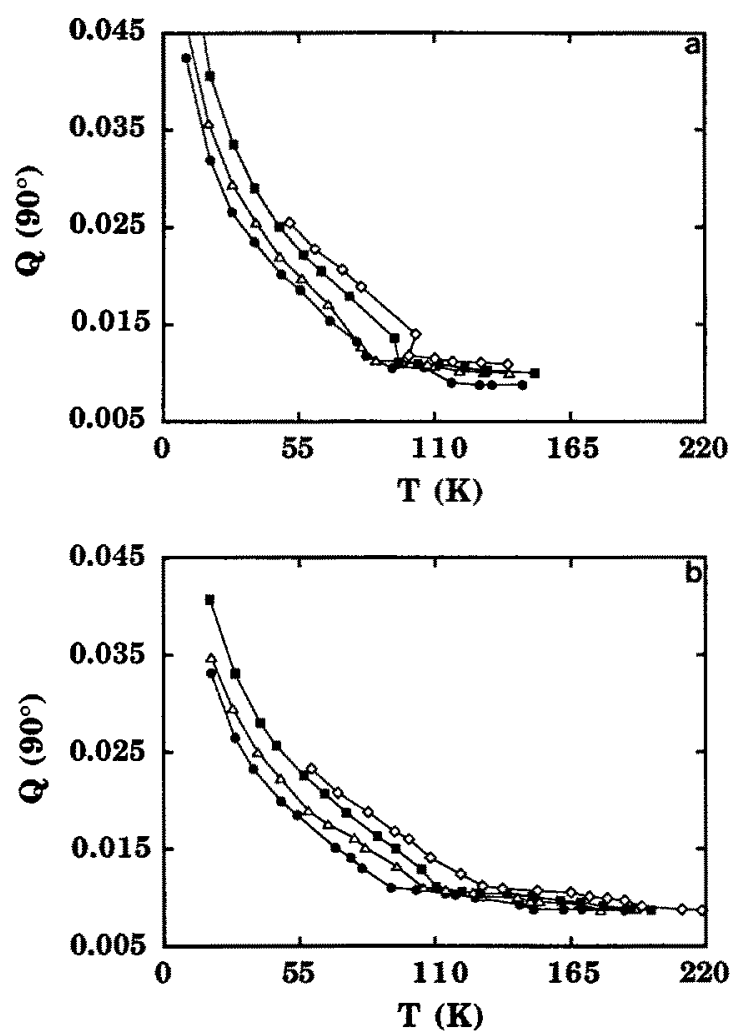

Fig. 6. Temperature dependence of peak heights of orientational angular distribution function $Q(\theta)$ at $q=90^{\circ}$ for the $N$-molecule clusters in cooling a and heating $\mathbf{b}$ runs. Filled circles, $N=100$; triangles, $N=150$; filled squares, $N=250$; diamonds, $N=350$
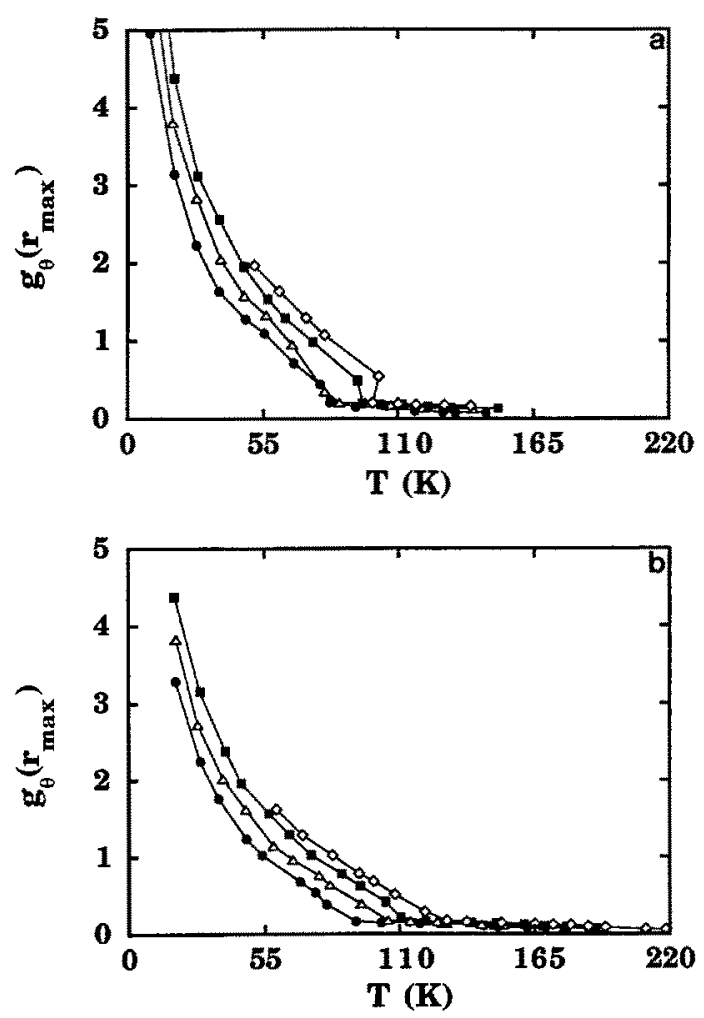

Fig. 7. Temperature dependence of heights of the first peak of the orientational pair-correlation function $g_{\theta}(r)$ for the $N$-molecule clusters in cooling a and heating $\mathbf{b}$ runs. Filled circles, $N=100$; triangles, $N=150 ;$ filled squares, $N=250$; diamonds, $N=350$ 

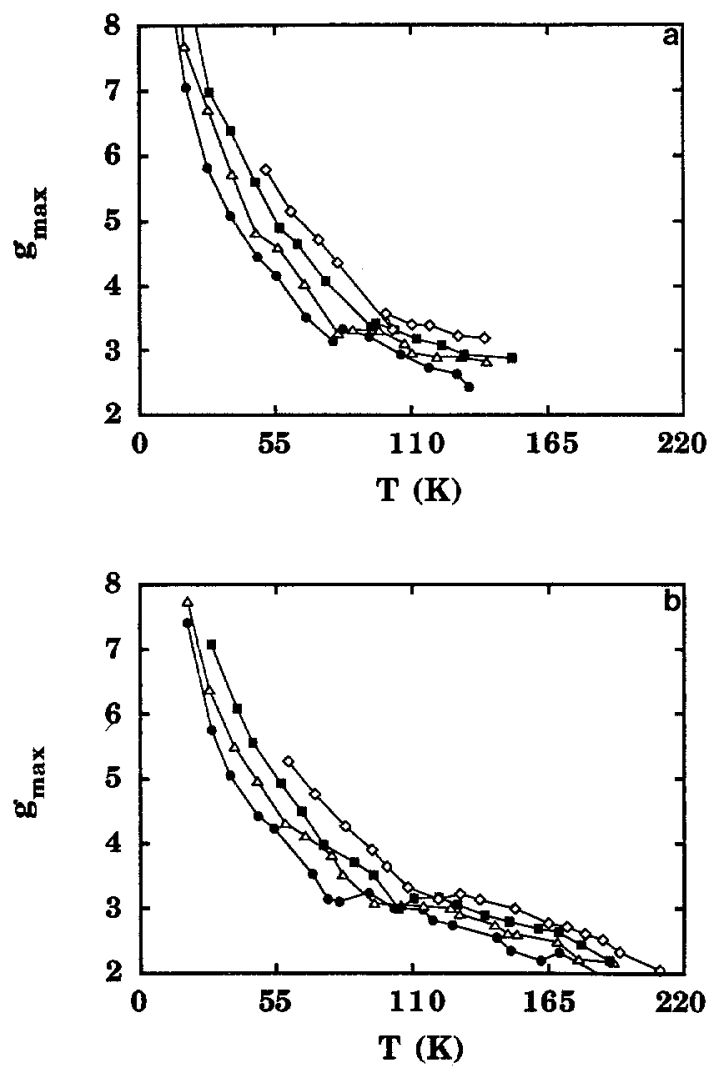

Fig. 8. Temperature dependence of heights of the first peak of the conventional pair-correlation function $g(r)$ for Te atoms in the $N$ molecule clustes in cooling a and heating b runs. Filled circles, $N=100$; triangles, $N=150$; filled squares, $N=250$; diamonds, $N=350$

Table 1. Rotational melting temperatures, in $\mathrm{K}$, for $N$-molecule clusters of $\mathrm{TeF}_{6}$ according to various indicators, and comparison with temperatures of transition from monoclinic to body centered cubic phase

\begin{tabular}{llrll}
\hline Indicator & \multicolumn{3}{l}{$N$} & \\
\cline { 2 - 5 } & 100 & 150 & 250 & 350 \\
\hline$D_{\theta}$ (indiv) & 82 & 95 & 105 & 113 \\
$\delta_{\theta}$ (indiv) & 86 & 97 & 106 & 113 \\
$\delta_{\theta}$ (mean) & 89 & 100 & 107 & 120 \\
$Q\left(90^{\circ}\right)$ & 79 & 95 & 104 & 116 \\
$g_{\theta}\left(r_{\max }\right)$ & 83 & 98 & 108 & 120 \\
$\begin{array}{l}\text { average of } \\
\quad \text { above }\end{array}$ & 84 & 97 & 106 & 116 \\
$\begin{array}{c}\text { monoclinic to } \\
\text { bcc }\end{array}$ & 81 & 95 & 106 & 111 \\
\hline
\end{tabular}

a From [19]

sensitive the results are to the chosen thresholds. The one upon which all of the others were based is the rotational diffusion coefficient. A change of $25 \%$ in the threshold value of $D_{\theta}$ selected shifts the characteristic rotational melting point by about $5 \mathrm{deg}$, or about $10 \%$ of the temperature spread over which the transition takes place in the simulations. Reducing by $25 \%$ the value of the rotational Lindemann index chosen as a threshold reduces the implied transition temperature by 10 to $15 \mathrm{deg}$. The other two indices, $Q\left(90^{\circ}\right)$ and $g_{\theta}\left(r_{\max }\right)$ are smooth, reproducible functions of the temperature (except during cooling when chance nucleation occurs) but their comparatively lower slopes make them perhaps half as sensitive as the orientational Lindemann index as gauges of the transition.

\section{Discussion}

The foregoing results show that all of the diagnostic functions can serve as indicators of rotational melting, and apparently serve as well for one cluster size as another. Rotational melting was found to occur long before translational melting. What the character of the rotational transition is can be inferred at once from the results summarized in Table 1. Rotational melting temperatures of the clusters are indistinguishable from the temperatures reported in a prior study [19] for the transition from the monoclinic phase to the bcc phase. Therefore, it is concluded that the two transitions are the same. This equivalence arose naturally, not having been forced while carrying out the analyses. Entirely different indicators had been used to recognize the monoclinic-bcc transitions in the clusters, including caloric curves, the translational Lindemann index, $\delta(t)$ [22], and Pawley-Fuchs projections [10].

Before describing the transition in more detail, it is worthshile to comment upon several aspects of the results found in this study. First, in those figures presenting results for both cooling and heating stages, it is apparent that the transitions occurre at lower temperatures during cooling runs than during heating. This difference is due to the fact that, if a transition is to happen during cooling, a nucleation event is essential, an event always seen to occur in the interior of a cluster. Such an event is a chance process mediated by kinetics, that requires supercooling. On the other hand, transitions during heating always occurred at the surface where the greater molecular freedom sufficed to provide the precursor of the warmer phase so that no stochastic nucleation event seemed to be needed. For this reason, temperatures of transition obtained in heating stages are more reproducible and more nearly thermodynamic in nature.

Next, it is evident that the smaller the cluster, the lower the transition temperature. In fact, as shown elsewhere [19], the temperature of the monoclinic-bcc transition decreases linearly with the reciprocal of the cluster radius. Such a trend is a consequence of the conventional capillary theory of cluster equilibrium [22-29] but compliance with the trend is not a confirmation of the validity of the capillary theory. On the contrary, results of the MD simulations show that the capillary theory is seriously deficient in its predictions about details of how the transitions evolve $[19,26]$. It makes the transitions far sharper functions of temperature than they are found to be in MD computations.

It is worthwhile to review briefly the nature of the transition from the body centered cubic phase to the monoclinic. As has been explained in detail elsewhere for 
the closely related system of $\mathrm{SF}_{6}[5,30]$ the spatial distributions of molecular sites in the two phases are similar, a circumstance obviously related to the fact that the transition is so facile that it can be seen on the extremely short time scale of MD simulations. In the warmer bcc phase, with all molecular axes parallel to the crystallographic axes (except for disorder), next-nearest molecules direct their fluorine atoms toward each other. This leads to steric distress which is relieved by the orientational disordering which is responsible for the large observed orientational Lindemann index and the large rotational diffusion that is found. In the transition to the monoclinic phase $1 / 3$ of the molecules rotate by $60^{\circ}$, allowing fluorines on one molecule to fit into the hollows of their neighboring molecules $[8,30]$, leading to lower potential energies, lower entropies, and to considerably greater orientational ordering and lower rotational diffusion rates.

The equivalence between rotational melting and the transition to a cubic phase found in the present investigation is in conformity with the empirical behavior of systems of globular molecules seen in neutron diffraction studies, as noted by Leadbetter and Lechner [21]. Indeed, in reviewing NMR observations of plastically crystalline systems, Boden [31] explicitly associates rotational melting with "the detailed changes in the reorientational motion at the ordered solid-plastic phase transition." In assessing the field, Leadbetter and Lechner commented that "the most interesting question is perhaps to what extent the rotational motion is isotropic." In the present simulations it was found, as expected, that the rotational coefficients of diffusion about all three axes were the same in the cubic phase. They were almost identical in the monoclinic phase, as well. Diffusion was accompanied by strongly damped librations about all axes, as can be inferred from the development of $\left\langle(\Delta \theta)^{2}\right\rangle$ with time. The extent to which diffusion about the 3-fold axis differed from that about the 4-fold axis has not yet been analyzed, however. The present simulations also confirmed in detail the reorientational mechanism of the bcc-monoclinic transition described in the preceding paragraph in which rotational jumps about the 3 -fold axes are the ratelimiting step. In the monoclinic phase there are two distinguishable orientations, denoted as $A$ for those $2 / 3$ of the molecules retaining the bcc orientations, and $B$ for the 1/3 which have changed orientation [8]. In all shells of all four monoclinic clusters studied the molecules in $A$ sites had smaller rotational diffusion coefficients and rotational Lindemann indices, by perhaps $30 \%$, than did those in $B$ sites. On the other hand, there was little difference between molecules in the two sites with respect to their translational diffusion coefficients and Lindemann indices $\delta$.

Even though our primary criterion for rotational melting was based on the substantial increase in rotational freedom accompanying the transition to a disordered phase, our computations reveal a small but significant rotational diffusion in the ordered phase even at temperatures as low as $50 \mathrm{~K}$. Although we are unaware of experimental measurements of molecular motions in monoclinic $\mathrm{TeF}_{6}$, NMR studies of the orthorhombic phases of $\mathrm{TeF}_{6}$ [32] and the very similar $\mathrm{MoF}_{6}$ [33] (both of which have packing efficiencies nearly the same as those of the monoclinic phases [20]) show that the molecules continue to execute rotations well below the bccorthorhombic transition temperature.

The present investigation employs several new procedures found to be useful in characterizing the orientational behavior of molecules in condensed phases. It illustrates how molecular dynamics simulations can play an important role in helping to interpret experiments. In particular, this study corroborates and amplifies the inferrences about molecular reorientations in extremely fast solid-state phase changes that had been drawn from electron diffraction studies of phase changes in supersonic jets [2]. In the next paper in this series the nucleation rates found in simulations will be compared with those derived by electron diffraction.

This research was supported by a grant from the National Science Foundation.

\section{Appendix}

\section{Definitions of diagnostic functions used in text}

Coefficients of rotational diffusion were determined for individual molecules as well as for clusters from the timeevolution of the mean-square orientational displacements $\left\langle[\Delta \theta(t)]^{2}\right\rangle$, where $\Delta \theta$ is the angle between the direction of one of the six bonds of a molecule (arbitrarily chosen) at time $t$ and the direction of the same bond at $t=0$. To reduce noise the average is taken over as many independent time origins as possible to improve the statistics. The mean-square displacement increases rapidly over the first picosecond or so as molecules undergo damped librations in the cages formed by their neighbors. This is followed by a steady increase corresponding to rotational diffusion. Coefficients of rotational diffusion

$$
D_{\theta}=\frac{1}{4} \frac{\mathrm{d}\left\langle[\Delta \theta(t)]^{2}\right\rangle}{\mathrm{d} t}
$$

were computed from the slope of the mean-square displacement over the period from 1 to $6 \mathrm{ps}$, with the factor $1 / 4$ arising from the 2 -dimensional character of the diffusion considered, namely that of the direction of a molecular axis.

In constructing the so-called orientational Lindemann index $\delta_{\theta}$, the variance in the intermolecular distance and the average linear repeat distance used in the conventional Lindemann index $\delta$ for crystals are replaced by the variance in the angles between bond directions and the angular separation taken to be the angle between two indistinguishable molecular orientations. Assigning a value of $90^{\circ}$ to the angular separation for octahedral molecules, we have, for a system of $N$ molecules each with $N_{b}$ bonds,

$$
\begin{aligned}
\delta_{\theta}= & \frac{2}{N(N-1) N_{b}^{2}} \sum_{i<j} \sum_{\alpha=1}^{N_{b}} \sum_{\beta=1}^{N_{b}}\left[\left\langle\left(\theta_{\alpha \beta}\right)_{i j}^{2}\right\rangle\right. \\
& \left.-\left\langle\left(\theta_{\alpha \beta}\right)\right\rangle^{2}\right]^{1 / 2}(\pi / 2)^{-1}
\end{aligned}
$$


where $\left(\theta_{\alpha \beta}\right)_{i j}$ is the angle between bond $\alpha$ in molecule $i$ and bond $\beta$ in molecule $j$. For comparison, one of the most commonly seen definitions of the Lindemann index $\delta$ is [22]

$\delta=\frac{2}{N(N-1)} \sum_{i<j}\left[\left\langle r_{i j}^{2}\right\rangle-\left\langle r_{i j}\right\rangle^{2}\right]^{1 / 2}\left\langle r_{i j}\right\rangle^{-1}$

where the similarity between (A2) and (A3) is evident, calling attention to conventions used in the definitions adopted. Although Lindemann originally intended for the ratio of standard deviation to mean interatomic distance to pertain to adjacent atoms, for clusters the ratio is more commonly taken over all interatomic distances as indicated in (A3). In our orientational case, the difference between the inclusion of only adjacent molecules and inclusion of all molecules was small. Therefore, we included all molecules in a cluster to reduce statistical noise.

Before defining the orientational pair-correlation function $g_{\theta}(r)$ it is helpful to introduce an auxiliary function $S_{i j}(r, n)$ that provides an index of how closely matched are the orientations of any two molecules $i$ and $j$ whose centers are separated by distance $r$. For hexafluorides the function can be taken to be

$S_{i j}(r, n)=\frac{1}{N_{b}} \sum_{\alpha=1}^{N_{b}} \sum_{\beta=1}^{N_{b}}\left[\left(\mathbf{n}_{\alpha i} \cdot \mathbf{n}_{\beta j}+1\right) / 2\right]^{n}$

where the unit vectors $\mathbf{n}_{\alpha i}$ and $\mathbf{n}_{\beta j}$ lie along the bonds $\alpha$ of molecule $i$ and $\beta$ of molecule $j$, and parameter $n$ controls the latitude tolerated in the matching. If parameter $n$ is greater than about 6 , the contribution $S_{i j}(\alpha, \beta)$ from any angle $\theta_{\alpha, \beta}$ is very nearly a Gaussian function of $\theta_{\alpha, \beta}$ with $\sigma_{\theta}^{2}=2 / n$. To obtain a standard deviation of $5^{\circ}$ in $\theta$, we took $n$ to be 265 .

The orientational pair-correlation function $g_{\theta}(r)$, then, is defined in terms of $S_{i j}(r, n)$ via the relation

$g_{\theta}(r)=\frac{v}{2 \pi r^{2}(N-1)}\left\langle\sum_{i<j} S_{i j}(r, n)\right\rangle$

where $v$ is the volume per molecule of the cluster, taken to be the same as that of the bulk in our calculations.

\section{References}

1. See, for example, reviews in: The plastically crystalline state. Sherwood, J.N. (ed.). New York: Wiley 1979
2. Dibble, T.S., Bartell, L.S.: J. Phys. Chem. 96, 8603 (1992)

3. Pawley, G.S.: Mol. Phys. 43, 1321 (1981)

4. Pawley, G.S., Thomas, G.W.: Phys. Rev. Lett. 48, 410 (1982)

5. Pawley, G.S., Dove, M.T.: Chem. Phys. Lett. 99, 45 (1983)

6. Dove, M.T., Pawley, G.S.: J. Phys. C.: Solid State Phys. 16, $5969(1983)$

7. Dove, M.T., Pawley, G.S.: J. Phys. C: Solid State Phys. 17, 6599 (1984)

8. Dove, M.T., Pawley, G.S.: Mol. Phys. 55, 1147 (1985)

9. Pawley, G.S., Brass, A.M., Dove, M.T., Refson, K.: J. Chim. Phys. 82, 249 (1985)

10. Fuchs, A.H., Pawley, G.S.: J. Phys. (Paris) 49, 41 (1988)

11. Boyer, L.L., Pawley, G.S.: J. Comput. Phys. 78, 405 (1988)

12. Torchet, G., de Feraudy, M.-F., Raoult, B., Farges, J., Fuchs, A.H., Pawley, G.S.: J. Chem. Phys. 92, 6768 (1990)

13. Beniere, F.M., Rousseau, B., Fuchs, A.H., de Feraudy, M.-F., Torchet, G.: In: Physics and chemistry of finite systems: from clusters to crystals, Vol. I, p. 363. Jena, P., Rao, B.K., Khanna, S.N. (eds.), Dordrecht: Kluwer 1992

14. Beniere, F.M., Fuchs, A.H., de Feraudy, M.-F., Torchet, G.: Mol. Phys. 76, 1071 (1992)

15. Rousseau, B., Boutin, A., Fuchs, A.H., Craven, C.J.: Mol. Phys. 76, 1079 (1992)

16. Bartell, L.S., Xu, S.J.: Phys. Chem. 95, 8939 (1991)

17. Bartell, L.S., Dibble, T.S., Hovick, J.W., Xu, S.: In: Physics and chemistry of finite systems: from clusters to crystals, Vol. I, p. 71. Jena, P., Rao, B.K., Khanna, S.N. (eds.). Dordrecht: Kluwer 1992

18. Bartell, L.S., Hovick, J.W., Dibble, T.S., Lennon, P.J.: J. Phys. Chem. 97, 230 (1993)

19. Xu, S, Bartell, L.S.: J. Phys. Chem. 91, 13550 (1993)

20. Kinney, K., Bartell, L.S. (unpublished research)

21. Leadbetter, A.J., Lechner, R.E.: In: The plastically crystalline state. Sherwood, J.N. (ed.). New York: Wiley 1979

22. Berry, R.S., Beck, T.L., Davis, H.L., Jellinek, J.: Adv. Chem. Phys. 70, part 2, 75 (1988)

23. Quirke, N., Sheng, P.: Chem. Phys. Lett. 110, 63 (1984)

24. Xu, S., Bartell, L.S.: J. Phys. Chem. 91, 13544 (1993)

25. Smith, W., Fincham, D.: CCP5 Program Documentation; The Program MDMPOL, SERC Daresbury Laboratory, Daresbury, U.K., 1982

26. Bartell, L.S., Chen, J.J.: Phys. Chem. 96, 8801 (1992)

27. Pawlow, P.: Z. Phys. Chem. 65, 545 (1909)

28. Buffat, P., Borel, J.-P.: Phys. Rev. A 13, 2287 (1976)

29. Reiss, H., Mirable, P., Whetten, R.L.: J. Phys. Chem. 99, 45 (1988)

30. Raynerd, G., Tatlock, G.J., Venables, J.A.: Acta Crystallogr. B38, 1896 (1982)

31. Boden, N.: In: The plastically crystalline state. Sherwood, J.N. (ed.). New York: Wiley 1979

32. Michel, J., Drifford, M., Rigny, P.: J. Chim. Phys. 67, 31 (1970)

33. Rigny, P., Virlet, J.: J. Chem. Phys. 51, 3807 (1969) 